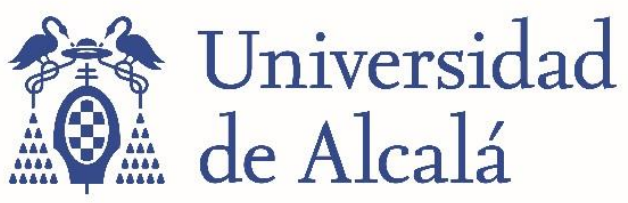

BIBLIOTECA

Document downloaded from the institutional repository of the University of Alcala: http://dspace.uah.es/

This is a published version of the following published document:

Escribano, F., López, L., Sanjuán, M.A.F.,"Analysis of Chaos-Based Coded Modulations under Intersymbol Interference", 2010, Journal of Computers, vol.5, no. 10 , pp.1459-1467.

Available at http://dx.doi.org/10.4304/jcp.5.10.1459-1467

C 2010 Academy Publisher

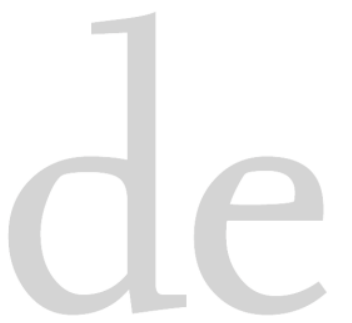

(Article begins on next page)

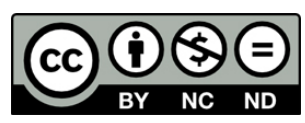

This work is licensed under a

Creative Commons Attribution-NonCommercial-NoDerivatives

4.0 International License. 


\title{
Analysis of Chaos-Based Coded Modulations under Intersymbol Interference
}

\author{
Francisco J. Escribano \\ Departamento de Teoría de la Señal y Comunicaciones, Universidad de Alcalá, 28805 Alcalá de Henares, Spain \\ Email: francisco.escribano@ieee.org \\ Luis López \\ Departamento de Sistemas Telemáticos y Computación, Universidad Rey Juan Carlos, 28933 Móstoles, Spain \\ Email: luis.lopez@urjc.es \\ Miguel A. F. Sanjuán \\ Departamento de Física, Universidad Rey Juan Carlos, 28933 Móstoles, Spain \\ Email: miguel.sanjuan@urjc.es
}

\begin{abstract}
Chaos-based communications systems have shown to be of potential interest in some kinds of dispersive channels. In this paper we analyze the behavior of a class of chaos-based coded modulations (CCM's) in channels with time-invariant intersymbol interference (ISI). We use the ISI distance spectrum of the CCM's to calculte bounds for the bit error rate (BER), and we provide the analytical condition a CCM has to comply to stand a limited quantity of ISI. We explain also the effect of the main modelling parameter of this class of chaos-based systems, and we show that the dynamics of the underlying chaotic map is in each case the main factor to account for the final performance. The results show that CCM systems are of potential interest in this kind of distorting environment.
\end{abstract}

Index Terms-Chaos, Intersymbol interference, Modulation coding, Error analysis

\section{INTRODUCTION}

The possibility of using chaotic signals to carry information was first considered in 1993 [1]. This aroused a big deal of work on chaotic communications, which became a hot topic in both nonlinear science and engineering. The interest in chaotic communications was due to the foreseen good properties of the chaotic signals in the fields of secure systems or broadband multiple access systems. In the case of secure systems, one could take advantage of the uncorrelation and unpredictability of the chaotic signals to build encryption algorithms. These are the same properties desirable for the spread sequences of a code division multiple access (CDMA) system. On the other hand, chaotic modulations and channel encoders

This paper is based on "Effects of Intersymbol Interference on Chaos-Based Modulations," by Francisco J. Escribano, Luis López, and Miguel A. F. Sanjuán, which appeared in the Proceedings of the 2nd International Conference on Signals, Circuits \& Systems (SCS), Hammamet, Tunisia, November 2008.

This work was supported in part by the by the Spanish Ministry of Education and Science under Project FIS2006-08525, by the Spanish Ministry of Science and Innovation under Project FIS2009-09898 and Project PET2008-0128, by the Spanish Ministry of Industry under Project TSI-020110-2009-103, and by Comunidad de Madrid under Project S2009TIC-1692 (CLOUDS). derived from chaotic systems attracted much attention, but the interest on this kind of chaotic communications dropped somewhat due to the bad performance of the systems proposed so far. In fact, they did not outperform other usual coded communication schemes, and they did not have even better performance than uncoded systems [2]-[5].

However, in later times and in some contexts we have witnessed the arising of some proposals with good performance as compared with classical communication systems [6]. Other recent proposals in the field make use of the fact that chaos based systems can be good for secure communications [7], [8]. This has reopened the trend of looking for efficient chaotic systems included in classical schemes where the potentiality of chaos in the channel could be properly exploited. Some chaos-based modulation systems working at the waveform level have already shown to be of potential use in multipath fading channels [9]. Other kind of chaos-based systems working at the coding level [10]-[12] have shown to provide good results in multiuser channels. It has been also shown that communications based on high dimensional chaotic systems and belief propagation decoding can offer an excellent performance characterized with thresholds [13].

Finally, some recent works stress the fact that, using chaos-based developments working jointly at the waveform and the coding level, it is possible to build multidimensional chaos-based coded modulations (CCM's) or concatenated systems based upon bad performing chaosbased coded modulations that, when employed in additive white Gaussian noise (AWGN) channels, can reach bit error rates (BER) comparable with other standard systems of similar complexity [6], [14]-[17]. Following one of this promising threads, recent studies have pointed out that chaos-based modulations working at this joint waveform and coding level can be of potential interest in frequencynon selective fading channels [18]. The key to this success resides in joining the fields of digital communications and chaos theory under a common framework. This 
makes possible the use of well known tools from the communications field in the evaluation and design tasks of chaos-based systems [17], [19]. Nevertheless, current literature lacks a thorough study showing what kind of channels could better match the properties of these CCM systems, since almost all the work has focused on pure AWGN or on multipath fading channels. On the other side, it is supposed that chaos-based signals in the channel should be appropriate in dispersive environments.

According to all this, in this article we address the task of showing that simple CCM systems that may perform worse than the most simple uncoded modulation in AWGN, can behave substantially better when the channel includes ISI. Thus, the chaotic signal can reveal its potential interest in broadband communications over frequency-selective time-invariant dispersive channels. We also derive bounds on the bit error probability and show that they can be tight enough to give reason of the behavior of this kind of systems. These principles can be easily extended to the whole kind of chaotic systems based on coded modulation, and help in the design and evaluation tasks, especially the condition we will establish for the existence of an error floor.

The comparison with a related standard trellis coded modulation (TCM) system [20] shows that CCM systems can keep the good properties of coded modulated signals in ISI channels. Note that our aim is not to provide methods to combat ISI impairment, but to show how CCM behave in this kind of channels and under what conditions they can be robust face to a limited level of ISI degradation. This impairment can easily appear in cable and radio systems due to the filters included at the transmitter side in order to comply with the band restrictions, or at the receiver side to reject off-band interferences [21]. Equalization is not always mandatory depending on the restrictions provided and on the margins available, so that in some systems it is to be expected a certain degree of controlled ISI.

The article is structured as follows. In Section II, the communications system model and the channel model are described with the needed detail. Section III is devoted to the calculation of the BER bounds. Section IV explains the effect of one of the key parameters of these systems. In Section V, we depict the simulation results together with the corresponding bounds. Section VI is devoted to the conclusions.

\section{SYSTEM DESCRIPTION}

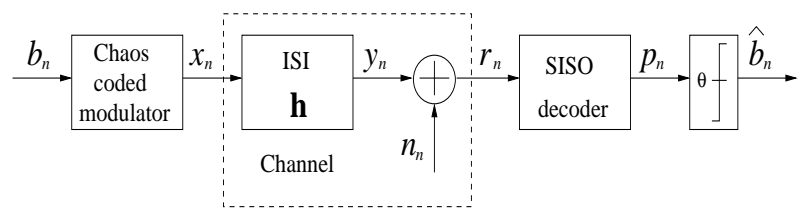

Fig. 1. Block diagram of the communications system.

In Fig. 1 we can see the scheme of the chaos-based communications system. The chaos-based coded modula-

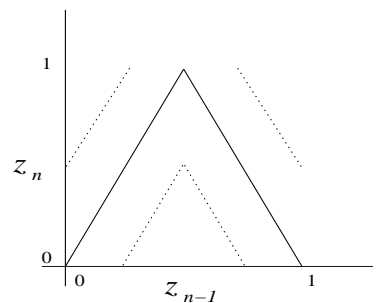

(a)

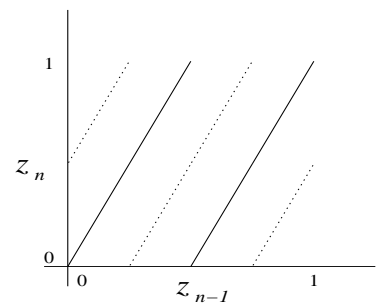

(b)
Fig. 2. Maps for the CCM systems. The continuous line corresponds to $f_{0}(\cdot)$; the dotted line, to $f_{1}(\cdot)$. (a) mTM (continuous line: TM); (b) mBSM (continuous line: BSM).

tion (CCM) system accepts as input an identically and independently distributed (i.i.d.) bit sequence $b_{n} \in\{0,1\}$, and it produces a chaos-based coded modulated sequence by means of a system relying on switched chaotic maps driven by small perturbations [6], following equations:

$$
\begin{aligned}
& z_{n}=f\left(z_{n-1}, b_{n}\right)+b_{n} \cdot 2^{-Q}, \\
& x_{n}=g\left(z_{n}\right)=2 z_{n}-1,
\end{aligned}
$$

where $f(\cdot, 0)=f_{0}(\cdot)$ and $f(\cdot, 1)=f_{1}(\cdot)$ are chaotic maps that leave the interval $[0,1]$ invariant. They are piecewise linear maps with slope \pm 2 wherever it is defined. The natural number $Q$ is a quantization factor that indicates the number of bits used to represent $z_{n}$ (and thus $x_{n}$ ). Note that the small perturbation manifests itself after $Q-1$ iterations. It has been shown that this kind of encoder leaves the set $S_{Q}=\left\{i \cdot 2^{-Q} \mid i=0, \cdots, 2^{Q}-1\right\}$ of $2^{Q}$ points invariant [6], so that, when taking as initial condition a value within this set (e.g. $\left.z_{0}=0\right), z_{n}$ can only take values from $S_{Q}$. In this way, we get a quantized chaotic sequence over $2^{Q}$ possible values that can be described as a trellis encoded sequence, with a state given by a shift register of $Q$ positions and two possible transitions determined by the input bit $b_{n}$.

We shall consider the following pairs of maps $f_{0}(\cdot)$ and $f_{1}(\cdot)$ :

1) Bernoulli shift map (BSM),

$$
f_{0}(z)=f_{1}(z)=2 z \bmod 1 .
$$

2) Tent map (TM),

$$
f_{0}(z)=f_{1}(z)=\left\{\begin{array}{ll}
2 z & 0 \leq z<\frac{1}{2} \\
2-2 z & \frac{1}{2} \leq z \leq 1
\end{array} .\right.
$$

3) The BSM and a shifted version of the same (multiBernoulli shift map, mBSM),

$$
\begin{gathered}
f_{0}(z)=2 z \bmod 1 \\
f_{1}(z)= \begin{cases}2 z+\frac{1}{2} & 0 \leq z<\frac{1}{4} \\
2 z-\frac{1}{2} & \frac{1}{4} \leq z<\frac{1}{2} \\
2 z-\frac{3}{2} & \frac{3}{4} \leq z \leq 1\end{cases}
\end{gathered}
$$

4) The tent map and a shifted version of the same (multi-tent map, mTM),

$$
f_{0}(z)= \begin{cases}2 z & 0 \leq z<\frac{1}{2} \\ 2-2 z & \frac{1}{2} \leq z \leq 1\end{cases}
$$




$$
f_{1}(z)=\left\{\begin{array}{ll}
2 z+\frac{1}{2} & 0 \leq z<\frac{1}{4} \\
2 z-\frac{1}{2} & \frac{1}{4} \leq z<\frac{1}{2} \\
\frac{3}{2}-2 z & \frac{1}{2} \leq z<\frac{3}{4} \\
\frac{5}{2}-2 z & \frac{3}{4} \leq z \leq 1
\end{array} .\right.
$$

In Fig. 2 we have depicted the corresponding maps. As stated, these CCM systems, when restricted to $S_{Q}$, allow an equivalent representation in terms of a trellis encoder [22], closely related to a trellis coded modulation (TCM) system [6]. In Fig. 3, for example, we can see the equivalent trellis encoder structure for the mTM CCM.

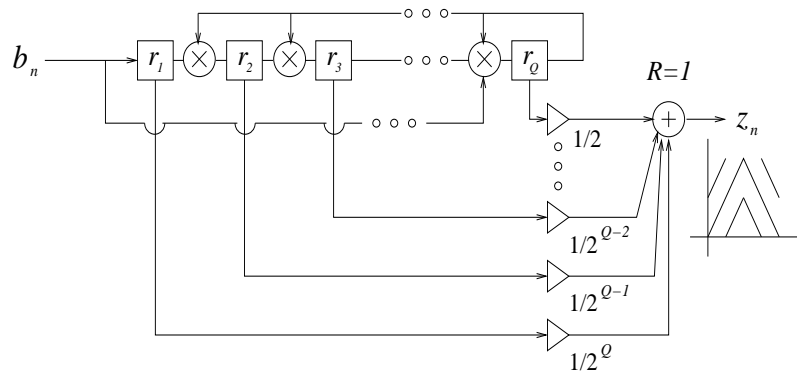

Fig. 3. Finite-state encoding structure for the mTM CCM.

The channel is an ISI channel with AWGN [21]. In digital communications, ISI impairment can easily appear due to the filters included at the transmitter side in order to comply with band restrictions, or at the receiver side to reject off-band interferences. As shown in Fig. 1 , the ISI model consists in a linear filter with finite impulse response $\mathbf{h}=\left(h_{-N}, \cdots, h_{N}\right)$, normalized to $\|\mathbf{h}\|^{2}=\sum_{m=-N}^{N}\left|h_{m}\right|^{2}=1$, so that it does not affect the signal power at the receiver. The AWGN process adds independent Gaussian samples $n_{n}$ with mean $\eta=0$ and power $\sigma^{2}$. We have considered two possible impulse responses: for low ISI, and for moderate ISI. These impulse responses are shown in Fig. 4, and they have $N=7$ coefficients. The parameter used to compare the degree of ISI is the ratio of signal power to interference power at the receiver, $\operatorname{SIR}=h_{0}^{2} /\left(\|\mathbf{h}\|^{2}-h_{0}^{2}\right)$ [21]. For low ISI we have $\mathrm{SIR}=14.84 \mathrm{~dB}$, and for moderate ISI, $\mathrm{SIR}=8.88 \mathrm{~dB}$.

Due to the trellis coded nature of this chaos-based signal, the receiver can be designed to decode the sequence using maximum likelihood (ML) or maximum a posteriori (MAP) sequence decoding algorithms. In this case, we have used a known MAP soft-input soft-output (SISO) decoder adapted to the decoding of this kind of chaotic sequences in AWGN channels [23], [24]. This SISO decoder, which has the advantage of allowing easy concatenation, is used here without any equalization, and thus it is simply based on the channel metrics of the AWGN case. Recall that we are interested in the effect of ISI in this kind of chaos-based coded modulations.

The SISO takes as input a block $\mathbf{r}$ of $M$ received samples,

$$
\begin{aligned}
& r_{n}=y_{n}+n_{n}=\sum_{m=-N}^{N} h_{m} x_{m+n}+n_{n}, \\
& n=0, \cdots, M-1,
\end{aligned}
$$

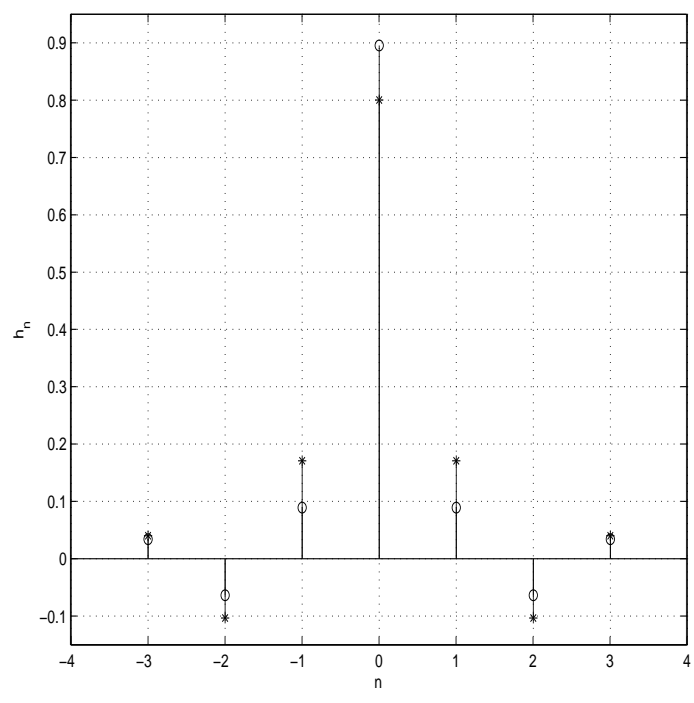

Fig. 4. Coefficients for the ISI FIR filters; 'o': low ISI; '*': moderate ISI.

and produces log probability ratios

$$
p_{n}=\log \left(\frac{P\left(b_{n}=1 \mid \mathbf{r}\right)}{P\left(b_{n}=0 \mid \mathbf{r}\right)}\right),
$$

which, when compared with the threshold $\theta=0$, generate the decoded sequence $\hat{b}_{n}$ (see Fig. 1).

\section{Performance ANALYSis}

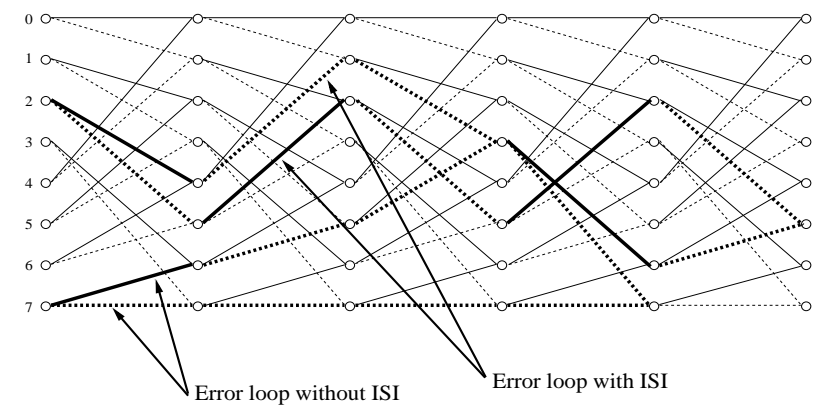

Fig. 5. Examples of minimal length error loops for the cases without ISI and with low ISI in the trellis representation of a BSM CCM with $Q=3$. Continuous lines: transitions given by input bit 0 . Dashed lines: transitions given by input bit 1 .

To establish comparisons with the performance of the chaos coded modulated systems, we will also take into account the case of uncoded binary phase shift keying (BPSK) over the same channel. The theoretical BER of BPSK in the ISI channel can be easily calculated if $N$ is not large [25]. With respect to the chaos coded modulated sequence, since the a priori probabilities for bits 1 and 0 are the same, MAP decoding will be equivalent to ML decoding and the pairwise error probability can be calculated as follows. There will be an error event when, having sent the chaos coded sequence $\mathbf{x}$, the decoder chooses a sequence $\mathbf{x}^{\prime} \neq \mathbf{x}$, where both sequences diverge at time $m$ and eventually merge again after $L$ steps in the trellis. Sequences $\mathbf{x}$ and $\mathbf{x}^{\prime}$ are thus related through a 
binary error event $\mathbf{e}=\mathbf{b} \otimes \mathbf{b}^{\prime}$ containing an error loop of length $L$ starting at time $m$ [26]. In the case of ML decoding, and taking into account that the metrics of the SISO decoder are calculated as a function of $\left(r_{n}-x_{n}\right)^{2}$ [23], this is equivalent to

$$
\sum_{n=m}^{m+L-1}\left(r_{n}-x_{n}^{\prime}\right)^{2}<\sum_{n=m}^{m+L-1}\left(r_{n}-x_{n}\right)^{2} .
$$

After some algebra, we get

$$
\begin{array}{r}
\sum_{n=m}^{m+L-1}\left[\left(y_{n}-x_{n}^{\prime}\right)^{2}-\left(y_{n}-x_{n}\right)^{2}\right]< \\
<2 \sum_{n=m}^{m+L-1}\left(x_{n}-x_{n}^{\prime}\right)^{2} n_{n} .
\end{array}
$$

For given $\mathbf{x}$ and $\mathbf{x}^{\prime}$, the right hand side member of inequality (11) is a Gaussian RV, so that the pairwise error probability can be calculated straightforwardly as

$$
P_{e}\left(\mathbf{x} \rightarrow \mathbf{x}^{\prime} \mid \mathbf{x}\right)=\frac{1}{2} \operatorname{erfc}\left(\sqrt{\frac{d_{\mathrm{ISI}}^{2} \frac{E_{b}}{4 P}}{N_{0}}}\right)
$$

where $P=1 / 3$ is the power of the chaos coded modulated signal ${ }^{1}$, and $d_{\text {ISI }}^{2}$ is an equivalent squared Euclidean distance in the ISI channel between $\mathrm{x}$ and $\mathrm{x}^{\prime}$ [27], given by

$$
d_{\mathrm{ISI}}=\frac{\sum_{n=m}^{m+L-1}\left(y_{n}-x_{n}^{\prime}\right)^{2}-\sum_{n=m}^{m+L-1}\left(y_{n}-x_{n}\right)^{2}}{d_{E}} .
$$

The factor $d_{E}^{2}=\sum_{n=m}^{m+L-1}\left(x_{n}-x_{n}^{\prime}\right)^{2}$ is the squared Euclidean distance between sequences $\mathbf{x}$ and $\mathrm{x}^{\prime}$.

According to (12), the error probability will be dominated for high $E_{b} / N_{0}$ by the error events leading to minimum values of $d_{\text {ISI }}^{2}$. The search for such error patterns may be a difficult task due to the structure of $d_{\mathrm{ISI}}^{2}$, since it depends on the ISI filter coefficients and on the CCM encoding trellis. Nevertheless, in the case of low or moderate ISI, where $y_{n}$ does not differ much with respect to $x_{n}$, the most probable error events are to be found among the error events with low loop lengths $L$. In Fig. 5 we can see two typical low length error loops for the BSM system with $Q=3$ in the case without ISI and with low ISI. Note that, since the CCM's are nonlinear and they do not meet the uniform error event property [28], the values of $d_{\text {ISI }}^{2}$ for a given binary error event $\mathbf{e}$ depend on the exact values of $x_{n}$ and $x_{n}^{\prime}$, and not only on e. This makes an important difference with respect to the study of related TCM schemes under ISI impairment, where uniform error properties and linearity properties can be extensively exploited [27].

As exemplified in Fig. 5, there is a difference between the typical error paths with and without ISI, which leads to a change in the distance spectrum of the chaos-based coded modulation. By performing some test simulations,

\footnotetext{
${ }^{1}$ In fact, this is the power when $Q \rightarrow \infty$ for the CCM's proposed, since they produce data uniformly distributed in $[-1,1]$. Nevertheless, it can be considered a good approximation for all the cases seen here.
}

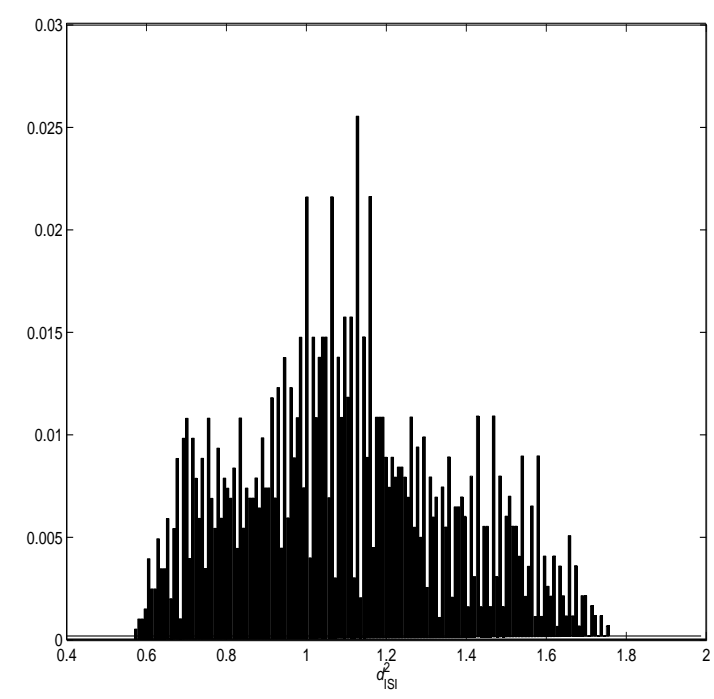

Fig. 6. Histogram of $d_{\text {ISI }}^{2}$ in the case of low ISI for the BSM CCM with $Q=4$ when the error events are of the kind described.

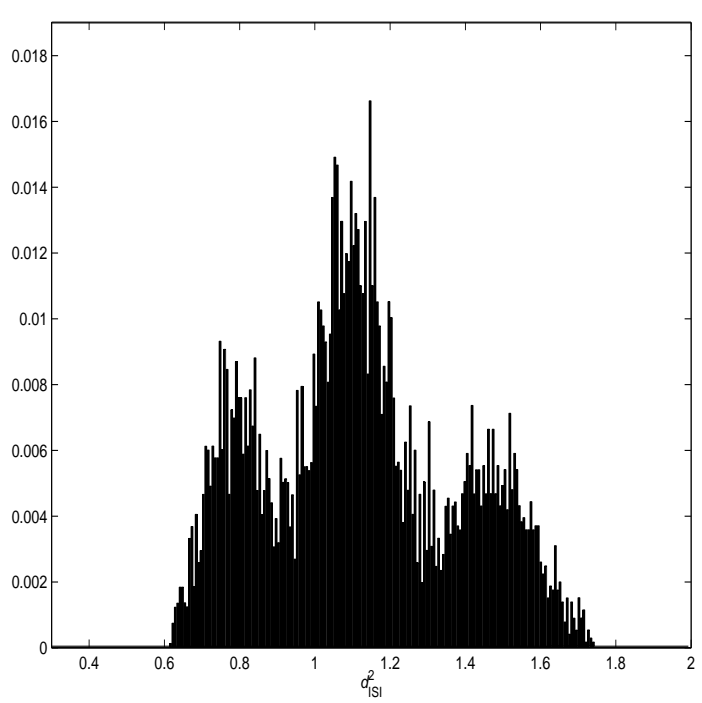

Fig. 7. Histogram of $d_{\text {ISI }}^{2}$ in the case of low ISI for the BSM CCM with $Q=5$ when the error events are of the kind described.

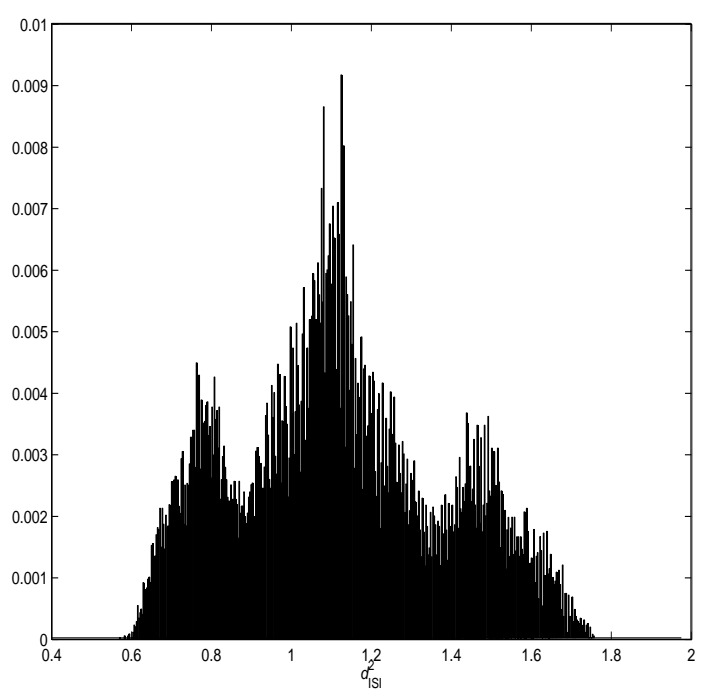

Fig. 8. Histogram of $d_{\text {ISI }}^{2}$ in the case of low ISI for the BSM CCM with $Q=6$ when the error events are of the kind described. 


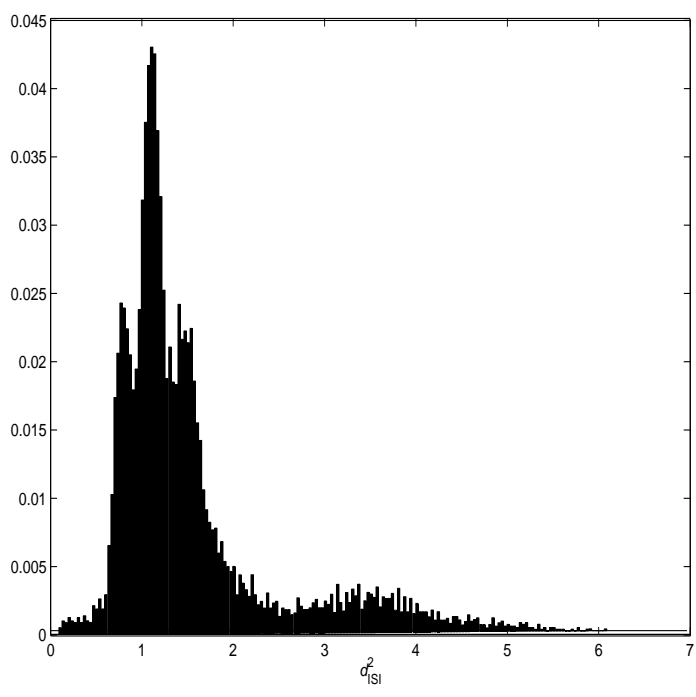

Fig. 9. Histogram of $d_{\text {ISI }}^{2}$ in the case of low ISI for the mTM CCM with $Q=5$ when the error events are of the kind described.

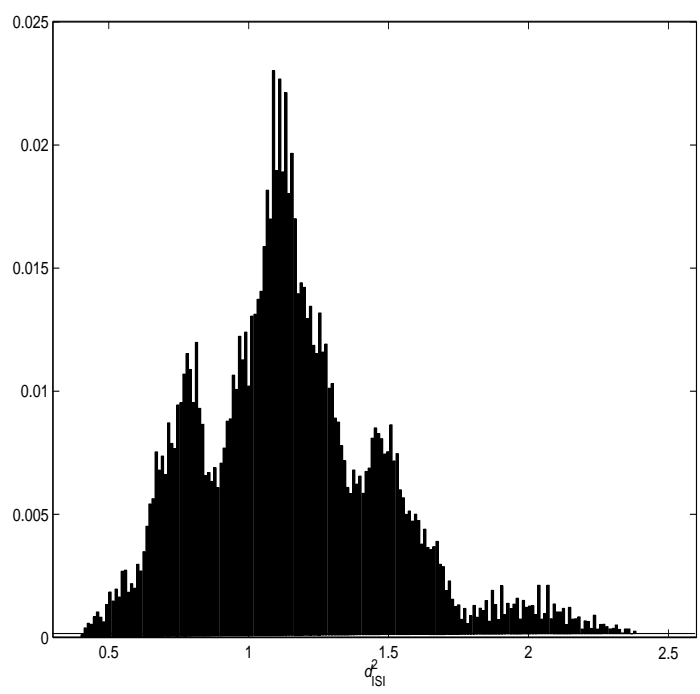

Fig. 10. Histogram of $d_{\mathrm{ISI}}^{2}$ in the case of moderate ISI for the BSM CCM with $Q=5$ when the error events are of the kind described.

it has been found out readily that the dominant error events in the low or moderate ISI cases are of the following kind as a function of the underlying map:

- For the BSM CCM, the most probable error events are those with Hamming weight $w(\mathbf{e})=2$, length $L=Q+2$ and structure $1,1,(Q) 0^{\prime} s$.

- For the TM CCM, the most probable error events are the same that give the minimum of $d_{E}^{2}$. They have length $L=Q+1$, Hamming weight $w(\mathbf{e})=Q$ and structure $1,(Q-2) 1^{\prime} s, 1,0$.

- For the mBSM CCM, these error events are of two kinds. The first kind is that with Hamming weight $w(\mathbf{e})=1$, length $L=Q+1$ and structure $1,(Q) 0^{\prime} s$ The second kind has Hamming weight $w(\mathbf{e})=2$, length $L=Q+3$ and structure $1,0,1,(Q) 0^{\prime} s$.

- For the mTM CCM, the error events with minimum $d_{\text {ISI }}^{2}$ are the same as for the TM CCM.
As stated before, the $d_{\text {ISI }}^{2}$ spectrum given by such error events depends on the ISI coefficients and on $x_{n}$ and $x_{n}^{\prime}$. In Figs. 6, 7, 8, we can see the histograms of the values of $d_{\text {ISI }}^{2}$ associated to the mentioned error events in the case of the BSM CCM with low ISI for $Q=4,5,6$. It is easy to verify that the number of possible sequences $x_{n}$, $x_{n}^{\prime}$ related through the binary error event $\mathbf{e}$ that can yield different values for $\sum_{n=m}^{m+L-1}\left(y_{n}-x_{n}^{\prime}\right)^{2}-\sum_{n=m}^{m+L-1}\left(y_{n}-\right.$ $\left.x_{n}\right)^{2}$ (and thus for $d_{\text {ISI }}^{2}$ ) is $2^{4 N+Q+L}$, so that we can evaluate exactly the associated distance spectrum for $d_{\text {ISI }}^{2}$ when $N, Q$ and $L$ are low enough. If not, it is always possible to estimate the related histogram by taking a significant number of samples after generating sets of test values for $x_{n}$ and $x_{n}^{\prime}$. Note that the spectra for the BSM CCM with low ISI and different $Q$ are quite similar, and this will lead to similar results both for the bound and for the BER results. We will see the reason for this insensibility to $Q$ in the next section.

On the other side, we have depicted the histogram of the associated distances for the mTM CCM with $Q=5$ (see Fig. 9). Though there is a quantity of distances with high values, we can see that there is a significant concentration of low values, and, since their minimum is lower than in the cases of the BSM CCM seen, we can foresee worse BER results with the MTM CCM than with the BSM CCM. We will verify this in Section V. In Fig. 10, we have depicted the histogram for the BSM CCM with $Q=5$ and moderate ISI. As could be expected, the distance spectrum broadens and we witness the appearance of distance values lower than the minimum attained in the case with low ISI, so that, accordingly, we will see in Section $\mathrm{V}$ how the bound and the BER will exhibit a corresponding degradation.

As verified throughout the last plots, in the cases considered here the values of $N, Q$ and $L$ are not high and the evaluation of all the possible values of $d_{\text {ISI }}^{2}$ for the mentioned binary error events is feasible. Let $D_{\mathbf{e}}$ denote the set of all such $d_{\text {ISI }}^{2}$ values for a given CCM, a given ISI FIR filter and the corresponding most probable binary error event, and let $D$ denote the number of elements in $D_{\mathbf{e}}$. Then, taking into account the expression for the error event probability (12), an average bound on the bit error probability can be calculated as [29]

$$
P_{b} \approx \frac{w}{2 D} \sum_{d_{\mathrm{ISI}}^{2} \in D_{\mathbf{e}}} \operatorname{erfc}\left(\sqrt{\frac{d_{\mathrm{ISI}}^{2} \frac{E_{b}}{4 P}}{N_{0}}}\right),
$$

where $w$ is the Hamming weight of the dominant binary error event e (i.e.: 2 in the case of the BSM CCM, and $Q$ in the case of the TM and mTM CCM's). In the mBSM CCM case, we have two possible error events and we have to average over the two contributions, one with $w=1$ and the other with $w=2$.

Nevertheless, we will verify that this bound does not always give reason of the bit error probability behavior, even if the binary error events actually happening are of the kind mentioned. In fact, we will verify the appearance of an error floor, which can be explained looking into the 
expression (11). If the differences $\left(x_{n}-x_{n}^{\prime}\right)^{2}$ are not high, and depending on the filter coefficients, there could exist the possibility that the right hand side of inequality (11) becomes negligible, specially when $E_{b} / N_{0}$ is higher than a threshold value (which, for fixed signal power $P$, means a vanishing value of the noise power $\sigma^{2}$ ). In this situation, inequality (11) becomes

$$
\sum_{n=m}^{m+L-1}\left(y_{n}-x_{n}^{\prime}\right)^{2}<\sum_{n=m}^{m+L-1}\left(y_{n}-x_{n}\right)^{2} .
$$

For a given set of filter coefficients $\mathbf{h}$, let us denote as $B_{\mathbf{e}}$ the number of pairs of sequences $\mathbf{x}, \mathrm{x}^{\prime}$ related through the binary error event $e$ that meet inequality (15). Since there is a total of $2^{4 N+Q+L}$ possible different values for $\sum_{n=m}^{m+L-1}\left(y_{n}-x_{n}^{\prime}\right)^{2}-\sum_{n=m}^{m+L-1}\left(y_{n}-x_{n}\right)^{2}$ when $\mathbf{x}$ and $\mathbf{x}^{\prime}$ are linked by a single error event $\mathbf{e}$ of the kinds stated, and since the related $\mathrm{x}$ sequences occur with equal probability for i.i.d. $b_{n}$ data, then the error floor associated with the binary error event e can be estimated as

$$
P_{b_{\text {floor }}} \approx \frac{w B_{\mathbf{e}}}{2^{4 N+Q+L}}
$$

where $w$ takes the mentioned values depending on the CCM kind and its dominant binary error event. Again, this calculation requires a limited value for $4 N+Q+L$, but there is always the possibility to bound this error floor by generating a sufficient number of test data. Note also that, for the mBSM CCM, there are two error events e and we should average over their corresponding contributions.

\section{EFFECT OF QUANTIZATION PARAMETER $Q$}

We have witnessed through Figs. 6, 7 and 8 a relative insensibility to $Q$ in the distance spectrum of the BSM CCM under low ISI. According to what was seen in previous section, the distance spectra exhibit a larger quantity of values when $Q$ grows, but they seem to keep the same shape, the same minimum and maximum values and the same structure. This has led us to foresee similar behaviors in point of BER, which in fact we will verify through simulation. But what is the reason for such insensibility?

First of all, let us focus on the BSM CCM without ISI. We see in Fig. 5 that the minimal error loop has length $L=Q+1$, and it corresponds to an error pattern of weight 1 , with structure $\mathbf{e}=(10 \cdots 0)$. Assuming that the bit on error is $b_{m}^{\prime}$ and the correct value is $b_{m}$, and according to equations (1) and (2), the correct $z_{m}$ sequence and the incorrect $z_{m}^{\prime}$ sequence start to diverge when

$$
\begin{gathered}
z_{m}=2 z_{m-1} \bmod 1+b_{m} \cdot 2^{-Q}, \\
z_{m}^{\prime}=2 z_{m-1} \bmod 1+b_{m}^{\prime} \cdot 2^{-Q},
\end{gathered}
$$

and their square difference in terms of the $x_{n}$ and $x_{n}^{\prime}$ sequences is

$$
\left(x_{m}-x_{m}^{\prime}\right)^{2}=4\left(z_{m}-z_{m}^{\prime}\right)^{2}=4 \cdot 4^{-Q}=4^{-Q+1},
$$

where we have made use of the fact that $\left|b_{m}-b_{m}^{\prime}\right|=$ 1. Since the rest of input bits $b_{m+1}, b_{m+2}, \cdots$ take the same values for both branches of the loop, the next square differences will only have to account for the effect of $b_{m}$ and $b_{m}^{\prime}$. Due to the expanding nature of the BSM map, in $z_{m+1}$ and $z_{m+1}^{\prime}$ the bits $b_{m}$ and $b_{m}^{\prime}$ will be weighted by $2^{-Q+1}$, so that the new square difference will be

$$
\left(x_{m+1}-x_{m+1}^{\prime}\right)^{2}=4 \cdot 4^{-Q+1}=4^{-Q+2} .
$$

Before the paths merge again in the loop, the last value for the square differences different from 0 will be associated with output symbols $z_{m+L-2}$ and $z_{m+L-2}^{\prime}$, so that

$$
\left(x_{m+L-2}-x_{m+L-2}^{\prime}\right)^{2}=4 \cdot 4^{-Q+L-2}=1 .
$$

Note that for the BSM CCM, this sequence of square differences associated to the error loop do not depend on the starting and ending states nor on the $b_{n}$ input values. This is not in general the case, and with the rest of CCM's these factors would have to be taken into account to calculate the spectrum of distances associated to their respective minimal error loops in the case without ISI. Note that the distance given by the mentioned error loop in the BSM CCM is

$$
\sum_{j=1}^{Q} 4^{-Q+j}=\frac{4}{3}\left(1-\frac{1}{4^{Q}}\right) .
$$

Though drawn for the BSM CCM, we have seen a general property for all the kind of CCM's based on expanding maps with slope \pm 2 : the partial square differences which contribute to the square distance associated to a given error loop have starting values weighted by $4^{-Q+1}$. The values of the subsequent differences from this value and on will depend on the given underlying map, the starting state of the loop and the input bit sequence, but they will be weighted in general by a factor $4^{-Q+j}$ growing from $4^{-Q+1}$ to a maximum value. Therefore, a growing $Q$ will have a vanishing effect on the calculations, since $4^{-Q+1}$ diminishes very fast with $Q$, and that is why the behavior of any of these BSM CCM's in AWGN will be almost the same from a minimum $Q$ value and on.

With respect to the ISI channel, we have seen in the previous section that the calculations of the ISI distances involve also accounting for other kind of error loops through the trellis (different to the ones typical for a pure AWGN channel), but they will exhibit the same property: increasing $Q$ and correspondingly the error loop length will have a limited effect in the final values from a minimum $Q$ value and on. That is why we can say that the properties of these CCM's in the ISI channel depend mainly on the underlying map and not on the parameter $Q$ which we introduced as an ad hoc development in order to build practical coded modulation schemes with an equivalent trellis representation.

\section{Simulation Results}

In Figs. 11 and 12 we can see the simulation results and the bounds for the several CCM's proposed under low and moderate ISI, respectively. We show also the theoretical bit error probability of BPSK in the same channels for 


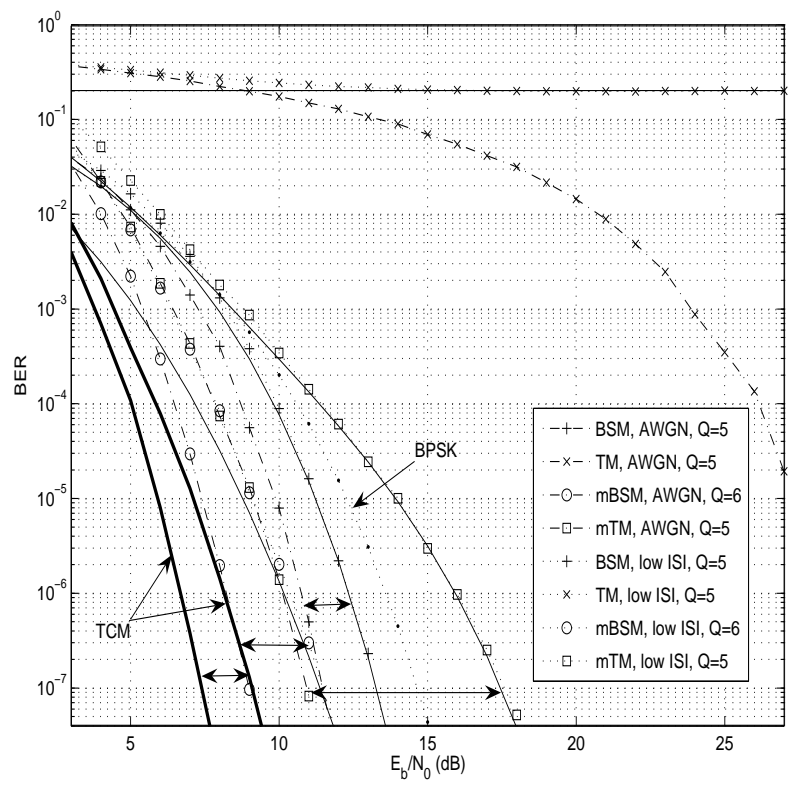

Fig. 11. Simulation results and bounds for AWGN and low ISI. Bounds are depicted with thin continuous lines. The performance of BPSK over the same ISI channel is depicted with a dotted line. The performance of the test TCM system is depicted with thick continuous lines, both for AWGN only (left) and for low ISI (right).

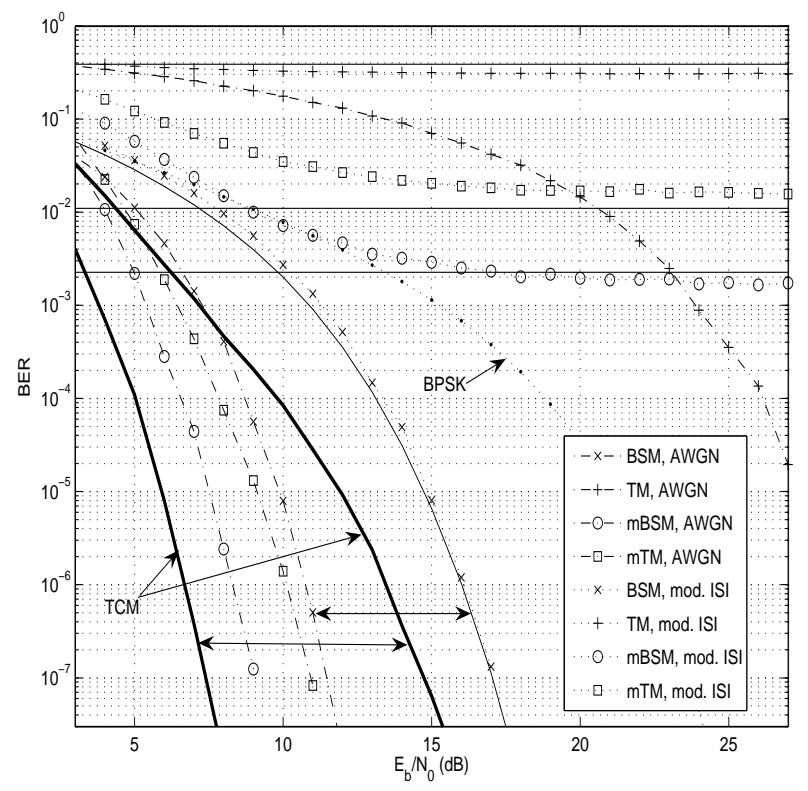

Fig. 12. Simulation results and bounds for AWGN and moderate ISI Bounds are depicted with thin continuous lines. The performance of BPSK over the same ISI channel is depicted with a dotted line. The performance of the test TCM system is depicted with thick continuous lines, both for AWGN only (left) and for moderate ISI (right). All the cases are for $Q=5$.

comparison [25]. We have depicted also the simulation results for a conventional $R=1 \mathrm{bit} / \mathrm{symbol}$ TCM scheme consisting on a constraint length $\nu=5$ encoder with generator polynomials 06 and 23 and quadrature phase shift keying (QPSK) modulation [29]. It is a rotationally invariant system suitable for dispersive channels. All the simulations have been run with data blocks of $M=10000$

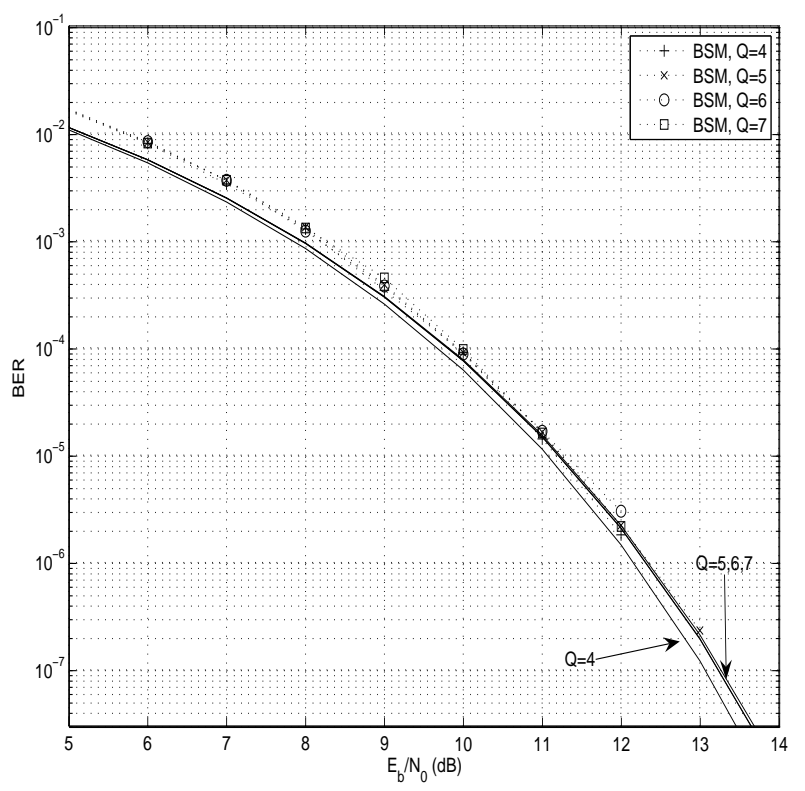

Fig. 13. Simulation results and bounds for low ISI in the case of the BSM CCM with different $Q$ values. The bounds are depicted with continuous lines.

bits and the BER results were recorded for 100 frames on error.

As stated for the CCM systems, in some of the cases the performance is dominated by condition (11) and the BER tends to zero as $E_{b} / N_{0}$ grows, while in other cases the mentioned error floor given by condition (15) is dominant for high signal to noise ratios. In low ISI, the TM CCM is severely affected by ISI impairment, while the rest of systems exhibit low or moderate losses. It is very significant that the mTM CCM comes next in $E_{b} / N_{0}$ loss in comparison with the AWGN channel, while the systems based on the Bernoulli shift map behave quantitatively and qualitatively better. When we have moderate ISI, however, only the BSM CCM is robust enough, keeping a steady coding gain with respect to the uncoded BPSK case, and the performance of the rest of CCM's degrades a lot, showing a high error floor and no coding gain. Note that in all the cases the corresponding bounds drawn from (14) and (16) are very tight and explain the BER behavior for high signal to noise ratios.

With respect to the conventional TCM system under low ISI, we can see that the loss in $E_{b} / N_{0}$ for a same BER of around $10^{-7}$ is similar to the loss in the case of the mBSM CCM, and slightly higher compared to the loss for the BSM CCM, while the MTM CCM is clearly worse (see the double arrows in Fig. 11). In the case of moderate ISI (see Fig. 12, double arrows), the BSM CCM outperforms the TCM system in point of $E_{b} / N_{0}$ loss. This gives a interesting hint of how this kind of chaos-based systems could exploit both the foreseen good properties of chaos in the channel and the already known possibilities of coded modulations in dispersive environments.

The cases leading to error floors are typical cases where equalization is absolutely mandatory [30]. The results obtained stress the fact that, if condition (15) is met with 
a given CCM for some low loop length error event in the ISI channel, then the BER tends fast to an error floor for growing $E_{b} / N_{0}$. In absence of equalization, this provides a design criterion for chaos-based coded modulations in situations where a low degree of unequalized ISI is to be expected. Note also how the systems based on chaotic maps with poor performing abilities in AWGN (due to its poor distance spectrum, like the TM CCM [31]) also lead to bad results in ISI.

In Fig. 13, we show the results and bounds in low ISI for the BSM CCM with different quantization levels. From a value of $Q=4$ and on, the behavior is the same, and the simulation results and the bounds are practically the same for $Q=5,6,7$. Thus, we verify that what was said in previous section about the negligible effect of $Q$ is in fact true so long as it has a value larger than a minimum. In this case, this minimum value is $Q=4$. This insensibility with respect to $Q$ is a desirable feature in chaos-based coded modulations, because the encoding and decoding complexity can be kept low enough without degrading the performance, while the dynamics of the case $Q \rightarrow \infty$ can be considered for evaluation and design purposes when necessary.

\section{Conclusions}

In this article we have analyzed the possibility to draw bounds for the bit error probability of a class of chaosbased coded modulations face to a limited amount of ISI. We have seen that, using the device of the ISI distance spectrum for a limited amount of possible error events, we can calculate simple bounds which are tight enough for high signal-to-noise ratios. By means of some examples, we have shown that chaos-based coded modulations of this kind can keep the good properties of coded modulations in dispersive environments [22]. We have as well verified the conditions for the appearance of error floors even in the presence of low ISI, and this provides a useful and interesting tool for design and evaluation tasks of related chaos-based coded modulations.

Moreover, we have analyzed the effect of the quantization parameter $Q$ on the final performance of the related systems, and we have seen that, so long as this parameter takes a value larger than a minimum of 4 , the behavior of the chaos-based coded modulation is in practice the same as for the ideal case with $Q \rightarrow \infty$. This links the performance results to the dynamics of the underlying map and gives a useful insight into chaosbased communications. At the same time, this allows the desing of practical systems with $Q$ as low as possible for bounded encoding and decoding complexity. All this opens a promising way for chaos-based communications since, as the principles shown here are directly applicable to the whole kind of chaos-based systems described by a trellis, they can help to cast a theoretical ground for new and successful developments in dispersive channels.

\section{REFERENCES}

[1] S. Hayes, C. Grebogi, and E. Ott, "Communicating with chaos," Phys. Rev. Lett., vol. 70, no. 20, pp. 3031-3034, May 1993.
[2] M. Hasler and T. Schimming, "Optimal and suboptimal chaos receivers," Proc. IEEE, vol. 90, no. 5, pp. 733-746, May 2002.

[3] F. C. M. Lau and C. K. Tse, Chaos-Based Digital Communication Systems. Berlin: Springer, 2003.

[4] F. J. Escribano, L. López, and M. A. F. Sanjuán, "Evaluation of channel coding and decoding algorithms using discrete chaotic maps," CHAOS, vol. 16, no. 1, pp. 013 103-0/16, March 2006.

[5] W. M. Tam, F. C. M. Lau, and C. K. Tse, Digital Communications with Chaos. Oxford: Elsevier, 2007.

[6] S. Kozic, T. Schimming, and M. Hasler, "Controlled one- and multi-dimensional modulations using chaotic maps," IEEE Trans. Circuits Syst. I, vol. 53, pp. 2048-2059, September 2006.

[7] J. Pizolato, M. Romero, and L. Goncalves-Neto, "Chaotic communication based on the particle-in-a-box electronic circuit," IEEE Trans. Circuits Syst. I, vol. 55, no. 4, pp. 1108-1115, May 2008.

[8] K. Wong and C. Yuen, "Embedding compression in chaos-based cryptography," IEEE Trans. Circuits Syst. II, vol. 55, no. 11, pp. 1193-1197, November 2008.

[9] Y. Xia, C. K. Tse, and F. C. M. Lau, "Performance of differential chaos-shift-keying digital communication systems over a multipath fading channel with delay spread," IEEE Trans. Circuits Syst. II, vol. 51, no. 12, pp. 680-684, December 2004.

[10] G. Mazzini, R. Rovatti, and G. Setti, "Chaos-based asynchronous DS-CDMA systems and enhanced rake receivers: measuring the improvements," IEEE Trans. Circuits Syst. I, vol. 48, no. 12, pp. 1445-1453, December 2001.

[11] G. Cimatti, R. Rovatti, and G. Setti, "Chaos-based spreading in DSUWB sensor networks increases available bit rate," IEEE Trans. Circuits Syst. I, vol. 54, no. 6, pp. 1327-1339, June 2007.

[12] G. Mazzini, G. Setti, and R. Rovatti, "Chip pulse shaping in asynchronous chaos-based DS-CDMA," IEEE Trans. Circuits Syst. $I$, vol. 54, no. 10, pp. 2299-2314, October 2007.

[13] S. Kozic and M. Hasler, "Belief propagation decoding of codes based on discretized chaotic maps," in Proc. of the IEEE Int. Symp. on Circuits and Syst. (ISCAS 2006), Kos, Greece, May 2006.

[14] F. J. Escribano, L. López, and M. A. F. Sanjuán, "Serial concatenation of channel and chaotic encoders," in Proc. of the 14th Int. Work. on Nonlinear Dynamics of Electronic Syst. (NDES 2006), Dijon, France, June 2006, pp. 30-33.

[15] F. J. Escribano, S. Kozic, L. López, M. A. F. Sanjuán, and M. Hasler, "Turbo-like structures for chaos coding and decoding," IEEE Trans. Commun., vol. 57, no. 3, pp. 597-601, March 2009.

[16] S. Kozic and M. Hasler, "Low-density codes based on chaotic systems for simple encoding," IEEE Trans. Circuits Syst. I, vol. 56, no. 2, pp. 405-415, February 2009.

[17] F.J. Escribano and L. López and M. A .F. Sanjuán, "Improving the performance of chaos-based modulations via serial concatenation," IEEE Trans. Circuits Syst. I, vol. 57, no. 2, pp. 448-459, February 2010.

[18] F. J. Escribano, L. López, and M. A. F. Sanjuán, "Chaos coded modulations over rayleigh and rician flat fading channels," IEEE Trans. Circuits Syst. II, vol. 55, no. 6, pp. 581-585, June 2008.

[19] - "Performance evaluation of parallel concatenated chaos coded modulations," Journal of Comm. Software and Syst., pp. 159-165, June 2008.

[20] G. Ungerboeck, "Channel coding with multilevel/phase signals," IEEE Trans. Inform. Theory, vol. 28, no. 1, pp. 55-67, January 1982.

[21] J. G. Proakis, Digital Communications. Boston: McGraw-Hill, Inc., 2001.

[22] J. B. Anderson and A. Svensson, Coded Modulation Systems. New York: Kluwer Academic / Plenum Publishers, 2003.

[23] F. J. Escribano, L. López, and M. A. F. Sanjuán, "Iteratively decoding chaos encoded binary signals," in Proc. of the 8th IEEE Int. Symp. on Signal Processing and Its Applications (ISSPA 2005), vol. 1, Sydney, Australia, August 2005, pp. 275-278.

[24] _ - "Exploiting symbolic dynamics in chaos coded communications with maximum a posteriori algorithm," Electron. Lett., vol. 42, no. 17, pp. 984-985, August 2006.

[25] V. Prabhu, "Intersymbol interference performance of systems with correlated digital signals," IEEE Trans. Commun., vol. 21, pp. 1147-1152, October 1973.

[26] J. Li, K. R. Narayanan, and C. N. Georghiades, "An efficient algorithm to compute the Euclidean distance spectrum of a general intersymbol interference channel and its applications," IEEE Trans. Commun., vol. 52, no. 12, pp. 2041-2046, December 2004. 
[27] C. B. Schlegel, "Evaluating distance spectra and performance bounds of trellis codes on channels with intersymbol interference," IEEE Trans. Inform. Theory, vol. 37, no. 3, pp. 627-634, May 1991.

[28] E. Biglieri and P. J. McLane, "Uniform distance and error properties of TCM schemes," IEEE Trans. Commun., vol. 39, no. 1, pp. 41-53, January 1991.

[29] S. Lin and D. J. Costello, Jr., Error Control Coding. Upper Saddle River, NJ: Pearson Prentice Hall, 2004.

[30] M. Ciftci and D. Williams, "Optimal estimation and sequential channel equalization algorithms for chaotic communications systems," EURASIP Jour. on Applied Signal Processing, vol. 4, pp. 249-256, December 2001.

[31] S. Kozic, K. Oshima, and T. Schimming, "Minimum distance properties of coded modulations based on iterated chaotic maps," in Proc. of the 11th Int. Work. on Nonlinear Dynamics of Electronic Syst. (NDES 2003), Scuol, Switzerland, May 2003, pp. 141-144.

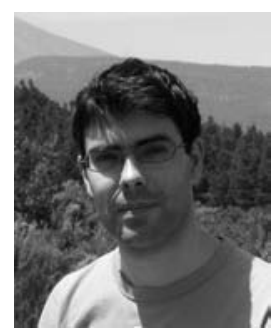

Francisco J. Escribano received his degree in Telecommunications Engineering at ETSITUPM, Spain, and his PhD degree at Universidad Rey Juan Carlos, Spain. He is currently Associate Professor at the Department of Signal Theory and Communications of Universidad de Alcalá, Spain, where he is involved in several undergraduate and master courses in Telecommunications. He has been Visiting Researcher at the Politectnico di Torino, Italy, and at the EPFL, Switzerland. His research activities are focused on Communications Systems and Information Theory, mainly on the topics of channel coding, modulation and multiuser detection, and on the applications of Chaos in Engineering.

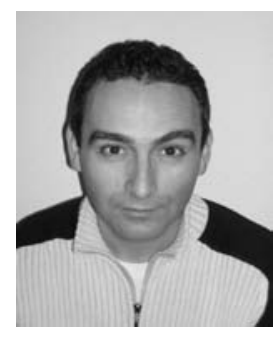

Luis López Fernández obtained his $\mathrm{PhD}$ in Computer Science at Universidad Rey Juan Carlos in 2003. He has a Telecommunications Engineering degree by the ETSIT-UPM and a Telecommunications Engineering degree by the ENST - Télécom Paris, both obtained in 1999. He is currently Associate Professor at Universidad Rey Juan Carlos, where he coordinates/collaborates in several undergradate and master courses in Computer Science and Telecommunications Engineering. His current research interests are concentrated on the applications of Complex Networks and Game Theory in the field of Computer Science in general, and IP networks in particular. Luis has coauthored more than 50 research publications in top scientific journals and conferences.

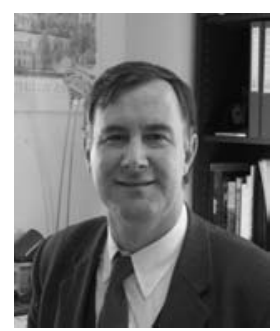

Miguel A. F. Sanjuán received his Degree in Physics from University of Valladolid, Spain in 1981, and a PhD Degree in Physics from National University at a Distance, Madrid, Spain in 1990. He was Visiting Research Associate at the Institute of Physical Science and Technology, University of Maryland, from July 1995 to September 1996 under the supervision of Prof. James A Yorke. He is a JSPS fellow and he was a Visiting Researcher at the University of Tokyo in February 2002, working in collaboration with Prof. Kazuyuki Aihara. Since 2002 he is Full Professor of Physics at the University Rey Juan Carlos, Madrid, Spain, where he is the Head of the Department of Physics and the Head of the Nonlinear Dynamics, Chaos and Complex Systems Group. In 2008, he was elected a Foreign Member of the Lithuanian Academy of Sciences. 